\title{
Contrast-enhanced ultrasound for the assessment of placental development and function
}

\author{
Victoria HJ Roberts*, 1 (iD) \& Antonio E Frias ${ }^{1,2}$ iD \\ ${ }^{1}$ Division of Reproductive \& Developmental Sciences, Oregon National Primate Research Center, Oregon Health \& Science University, Portland, OR 97006, USA; \\ ${ }^{2}$ Department of Obstetrics \& Gynecology, Oregon Health \& Science University, Portland, OR 97123, USA; *Author for correspondence: robertsv@ohsu.edu
}

BioTechniques 69: 393-399 (November 2020) 10.2144/btn-2020-0069

First draft submitted: 15 May 2020; Accepted for publication: 28 July 2020; Published online: 4 September 2020

\begin{abstract}
The use of contrast agents as signal enhancers during ultrasound improves visualization and the diagnostic utility of this technology in medical imaging. Although widely used in many disciplines, contrast ultrasound is not routinely implemented in obstetrics, largely due to safety concerns of administered agents for pregnant women and the limited number of studies that address this issue. Here the microbubble characteristics that make them beneficial for enhancement of the blood pool and the quantification of real-time imaging are reviewed. Literature from pregnant animal model studies and safety assessments are detailed, and the potential for contrast-enhanced ultrasound to provide clinically relevant data and benefit our understanding of early placental development and detection of placental dysfunction is discussed.
\end{abstract}

\section{Vascular remodeling during pregnancy}

The placenta is a unique and remarkable organ that must perform a multitude of functions to provide for the developing fetus while also supporting its own growth throughout pregnancy. It provides the functional interface between the growing baby and the mother, and aberrant placental development has been linked to many adverse obstetric outcomes. To appreciate the challenges of assessing the placenta in vivo, and to provide context for the advantages and disadvantages of various imaging techniques that can be applied to this objective, we must start with the fundamentals of uteroplacental vascular structure.

During pregnancy, the uterus undergoes extensive remodeling of the maternal vasculature, in addition to development of an entirely new circulatory system within the placenta and fetus. At the time of implantation, the conceptus must establish a strong foundation as it anchors to the wall of the uterus. Trophoblast cells migrate into the decidua and invade the maternal spiral arteries, converting them from tightly coiled high-resistance vessels to low-resistance, high-flow conduits [1,2]. Inadequate remodeling of the spiral arteries is the underlying cause of placental insufficiency with poor maternal supply failing to satisfy fetal demand, resulting in pathological outcomes such as preeclampsia, fetal growth restriction and stillbirth [3,4]. The vascular organization within the placenta is remarkable given that two separate vascular networks must develop in tandem: the maternal intervillous compartment as the source of nutrients and oxygen, and the fetal compartment structured in a complex arborized pattern of villi intended to maximize surface area available for the exchange of nutrients, oxygen and waste between the two separate circulations (Figure 1). The syncytiotrophoblast is the maternal-fetal exchange surface of the villi that functions as a transport and protective barrier. The uncompromised integrity and normal functioning of the syncytiotrophoblast layer is essential for pregnancy success and appropriate fetal development.

Much knowledge of placental function has been gained from post-delivery studies when the tissue is easily accessible for evaluation. However, these provide minimal insight into perfusion characteristics and offer no way to identify or intervene in ongoing pregnancies at risk for adverse outcomes. To address this, the recent $\mathrm{NIH}$-initiated Human Placenta Project has spurred a substantial research effort to expand the capabilities for real-time assessment of placental function in utero [5] with a focus on placental hemodynamics. Many advances have been made with a variety of imaging modalities and an expansion of this research area dominated by MRI methodologies, but also including ultrasound applications that extend beyond routine clinical evaluation of pregnancy. The focus of this review will be contrast-enhanced ultrasound for the assessment of placental development and hemodynamics.

\section{Basic principles of ultrasound}

Ultrasound technology relies on the acoustic detection of energy generated by a transducer that is attenuated and reflected by structures within the body, and returned to the transducer for conversion into a grayscale image [6]. The echogenicity of a tissue is a measure of acoustic reflectance with dense structures reflecting increased sound waves and appearing lighter on the resulting images. Typically, fluids lack echogenic properties, fail to produce a signal and appear black on standard B-mode ultrasound. Doppler ultrasound can be used to overcome this limitation by relying on the principle of changes in frequency of the backscatter signal from small moving structures, such as red blood cells. The difference between the transmitted and received frequencies is the Doppler shift. From this, blood flow velocity can be calculated if several other factors are known and remain consistent (for detailed review of Doppler principles, refer 


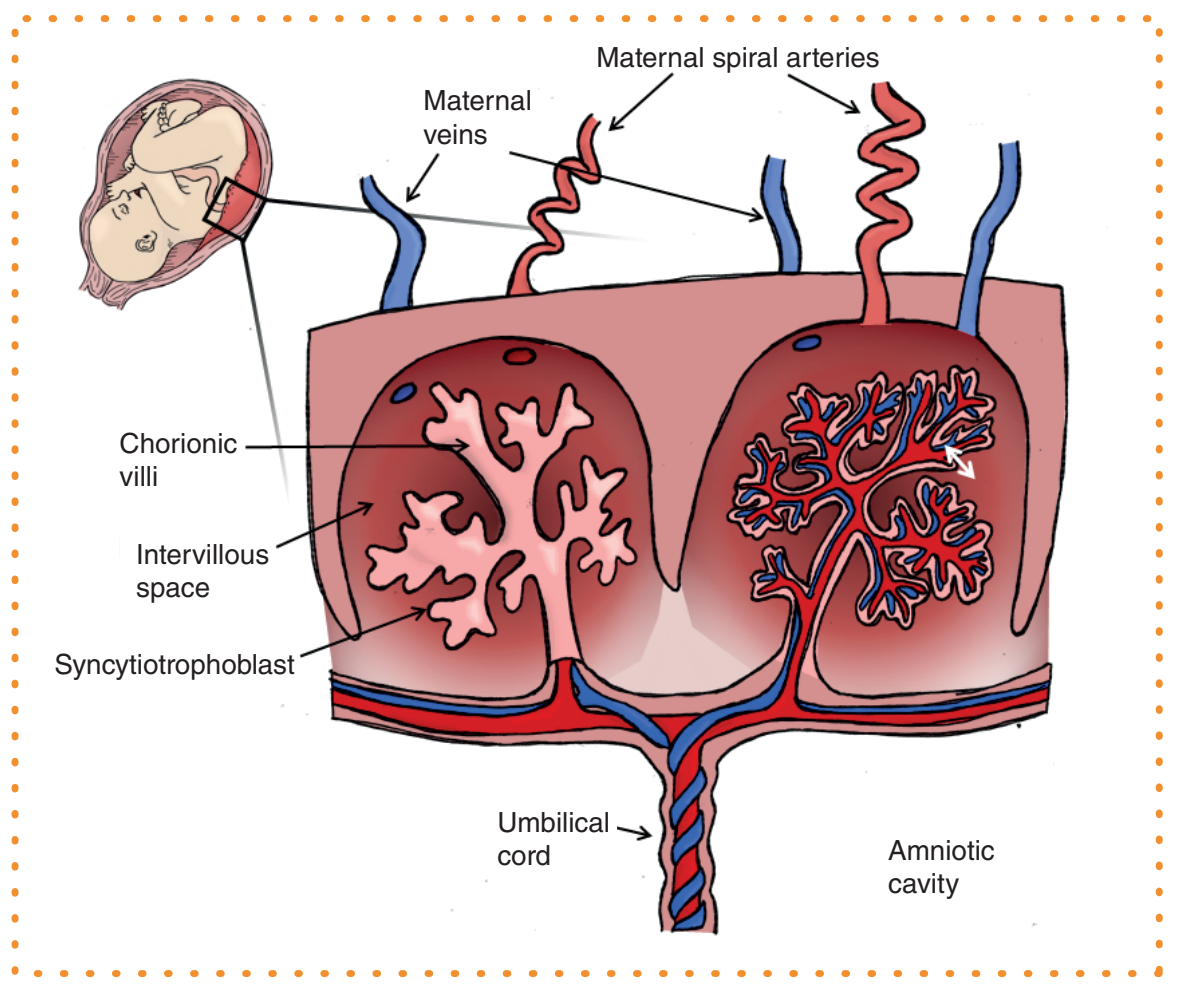

Figure 1. Schematic diagram of a cross-section through the placenta. Maternal blood arrives to the placenta through the spiral arteries to perfuse the intervillous space and drains through the maternal veins. Nutrient and waste exchange (white double-headed arrow) occurs across the syncytiotrophoblast layer from the maternal intervillous blood pool to the fetal capillaries within the chorionic villi. Nutrient-rich blood is transported to the fetus via the umbilical vein, and deoxygenated blood returns via the two umbilical arteries.

to the work of Merritt [7] and Moorthy [8]). Doppler ultrasound is routinely used in obstetrics to quantify uterine hemodynamics. Abnormal Doppler indices are indicative of abnormal vascular resistance, providing some diagnostic utility for pregnancies at risk for placental insufficiency [9,10]. Doppler has also been implemented for more detailed assessments of the maternal blood flow to the spiral arteries [11,12] with advancements in modeling and quantification made using 3D power Doppler [13,14]. As an alternative, ultrasound contrast agents can be used to enhance the acoustic signal from blood in standard B-mode imaging resulting in improved grayscale enhancement [15]. This methodology permits assessment of microvascular perfusion, and the use of contrast agents for signal enhancement during ultrasound has been utilized for several decades in medical imaging [16].

\section{Contrast agents}

The most commonly used contrast agents in ultrasound imaging are microbubbles. Their composition and content can vary, but microbubbles are typically composed of a gas core stabilized by an outer shell made of lipids, proteins or polymers [17]. Microbubble production has evolved over the years, and their composition and physiochemical properties can be tailored toward a specific functional application [18]. Enhanced stability of microbubble agents is a result of modification to the microbubble shell and/or gas content with biocompatible gases of high density and high molecular weight being less prone to outward diffusion, and newer encapsulation materials reducing surface tension [15,18], thus improving their efficacy.

The rheology of microbubbles has been assessed in intravital microscopy studies that demonstrated their unimpeded transit through the microcirculation of normal muscle beds with no evidence of aggregation, and with a similar velocity to red blood cells in arterioles, venules and capillaries [19]. They are typically less than 5 microns in diameter and remain within the intravascular space [17]. Importantly, microbubbles are metabolically inert [20], and they do not cause an immune response of the host [21]. Following intravenous administration, the microbubble gas content is exhaled via the lungs 10 to $15 \mathrm{~min}$ post injection, and the shell components are metabolized either by the liver or by kidney filtration and urine excretion [22].

As stated, signal enhancement in contrast ultrasound relies on the detection of acoustic energy produced by microbubbles within the vascular compartment. The rationale for using microbubbles is their compressibility under ultrasound where they undergo volumetric oscillations in which they are compressed during the pressure peaks and expand during the pressure nadirs [23]. The resulting microbubble vibrations generate strong backscattered acoustic signals and typically resonate at $1-10 \mathrm{MHz}$, which is the range of conventional ultrasound scanners used in diagnostic imaging. Their harmonic frequency produces a nonlinear reflection pattern at a low mechanical index (typically $<0.2$ ), resulting in an improved signal-to-noise ratio and enabling real-time perfusion imaging [18]. The efficiency of 
nonlinear excitation depends on the microbubble acoustic properties; data acquisition must be optimized in the ultrasound machine settings for each type/composition of the microbubble and standardized for consistency to produce good contrast enhancement. Commercially manufactured microbubbles have been available since the 1980s, and several US FDA approved formulations are available for diagnostic imaging. In pregnancy, Definity ${ }^{\circledR}$ and Lumason ${ }^{\circledR}$ microbubbles are designated as FDA category B, and Optison ${ }^{\circledR}$ is category C.

\section{Overview of safety considerations}

Concerns for the safety of contrast agent use in pregnant women provide the biggest obstacle to implementation of this technique in routine clinical assessment of at-risk pregnancies. The safety aspect has several components that must be considered: the adverse maternal effects, the potential damage to placental tissue integrity and the subsequent risk of disruption to fetal development.

Starting with potential effects of intravenous administration of contrast, data regarding side effects has been compiled from human clinical use in nonpregnant individuals. Earlier reports encouraged post-administration patient monitoring for signs of anaphylaxis and flank pain, but in 2007 the FDA published additional warnings of more serious cardiopulmonary reactions [24], which instigated an upsurge in safety and efficacy studies and a transient period of reduced and cautious contrast use. A series of safety reports followed (reviewed in Appis et al. [18]), in particular a large study of more than 78,000 administered doses of two commonly used, commercially produced agents, Definity ${ }^{\circledR}$ and Optison ${ }^{\circledR}$, which determined the rate of adverse reactions to be 0.01 and $0.0006 \%$, respectively, consistent with an anaphylactic reaction [25]. The overall consensus from the collective study outcomes was that severe adverse events are rare and the use of contrast agents remains highly beneficial as a diagnostic tool.

A study conducted in both a mouse model and a human patient cohort demonstrated that microbubbles are retained in the renal cortex because of complement-mediated interactions with the microvasculature of the glomerular endothelium [26]. These findings demonstrated that retained microbubbles did not interfere with renal perfusion, but they may provide an explanation for the flank pain that has been reported in some patients post contrast agent administration.

Importantly, it has been demonstrated that administration of microbubbles to the maternal vascular compartment does not negatively impact fetal vascular hemodynamics. Specifically, in a study of 69 pregnant women using the contrast agent Levovist ${ }^{\circledR}$ (galactose shelled microbubbles measuring $<8$ microns in diameter) in combination with Doppler ultrasound to examine uteroplacental circulation in late pregnancy, no change in umbilical artery blood flow velocity or fetal cardiac parameters was shown [27]. Similarly, use of Levovist ${ }^{\circledR}$ to examine twin-twin transfusion syndrome in 14 cases reported no fetal compromise [28]. These outcomes provide reassurance that the microbubbles do not enter the fetal circulation, which is not surprising given their size and charge properties. Notably, there were no deleterious effects on fetal growth or evidence of placental damage [27]. It is the potential placental damage and compromised syncytiotrophoblast integrity that needs to be satisfactorily addressed if contrast ultrasound and microbubble use is to be accepted for future routine obstetric practice. The mechanisms of ultrasound-induced cellular disruption are overviewed in the following.

\section{Microbubble destruction by ultrasound: mechanisms \& considerations for use}

For the purpose of this review, and for simplicity, the administration of contrast agents during ultrasound broadly speaking serves two functions: to aid visualization and identification of anatomical/flow-related abnormalities, or for quantification of tissue perfusion (Figure 2). Unlike an organ such as the heart where abnormal flow direction or an impeded valve may be readily apparent by enhancement alone, the complex architecture of the placenta is more challenging to interrogate. The movement of maternal blood within the intervillous space (Figure 1) bathing the villous tree can be visualized for observational assessment, but to generate quantitative metrics of perfusion requires analysis of microbubble destruction and replenishment in this vascular space. The imaging requirements needed to observe replenishment provide a powerful tool to examine microvascular perfusion but raise additional concerns about this imaging method due to the potential effects of cavitation.

Cavitation refers to the creation, vibration and collapse of small gas-filled bodies in a fluid [17]. This phenomenon can occur with ultrasound of fluids in the absence of any added agent. As gas-filled bodies, microbubbles act as nuclei by lowering the threshold for cavitation and increasing the possibility of damaging cavitation-related effects occurring [29]. Specifically, noninertial cavitation refers to the stable oscillation of bubbles under ultrasound at a low mechanical index where they vibrate at harmonic multiples of the transmitted ultrasound frequency and improve the acoustic signal. Inertial cavitation, typically at a higher mechanical index/acoustic pressure, causes microbubbles to vibrate asymmetrically and erupt violently, resulting in microbubble destruction (Figure 2) [30].

In real-time imaging, the intentional destruction of microbubbles in the acoustic field facilitates the visualization of replenishment of the vascular space to allow quantitative assessment of tissue perfusion over time. Data analysis requires the manual drawing of a region of interest over an individual perfusion domain [31], and the rate of replenishment of microbubbles from the time of destruction to maximal refilling is plotted in a replenishment kinetic curve [32]. The rate of replenishment in the region of interest reflects the microvascular blood flow velocity [23] and provides spatiotemporal quantification of tissue perfusion (Figure 3).

The potential adverse effects of microbubble destruction and the subsequent possibility of damage to the syncytiotrophoblast with altered permeability and compromised villous integrity raise concerns for altered placental function with use of contrast-enhanced ultrasound in pregnancy. There are several proposed mechanisms from which tissue damage results. Microbubbles under increased acoustic pressure create high-energy microstreams that increase shear stress on the membrane of endothelial cells and can result in 


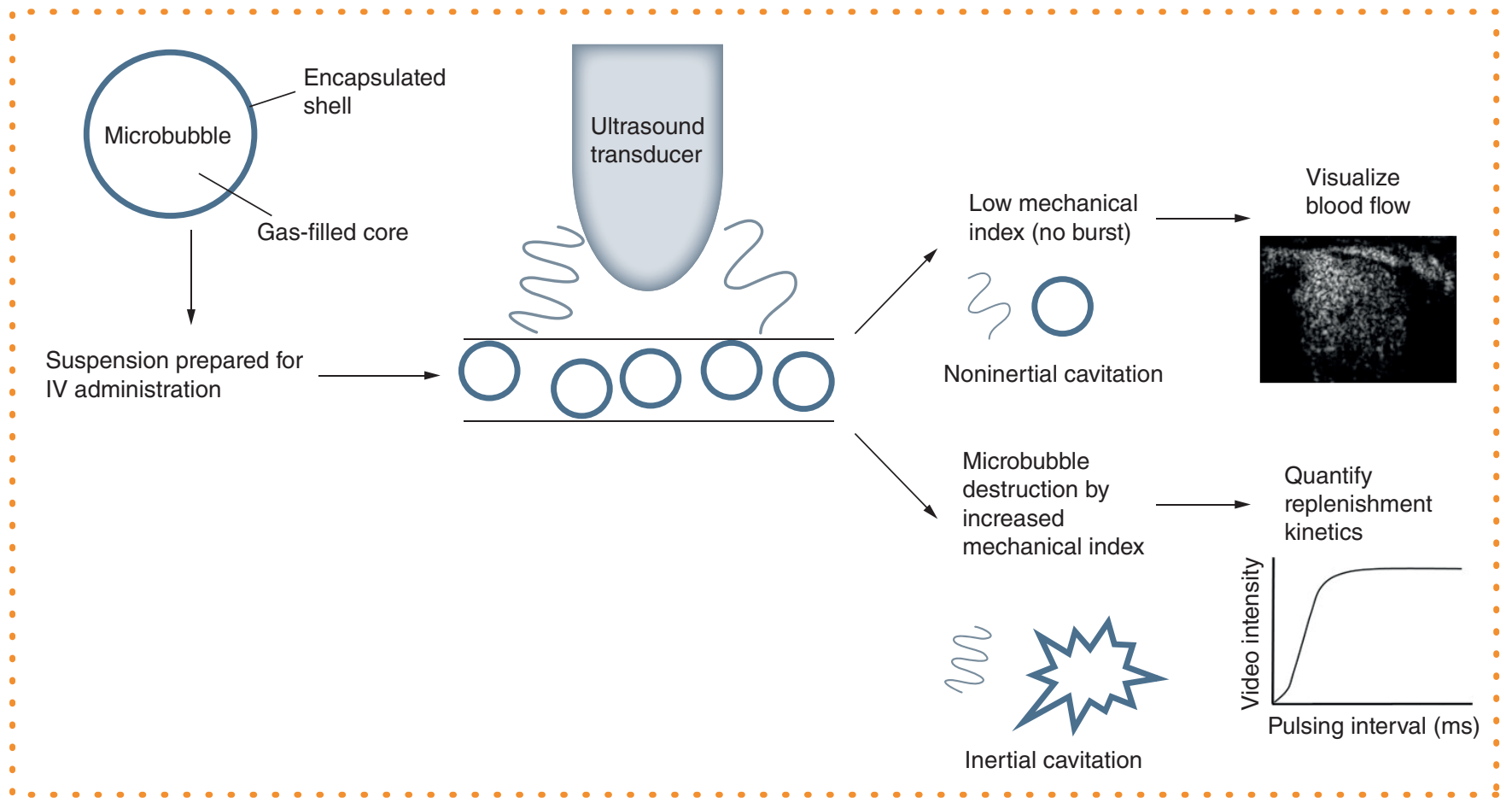

Figure 2. Contrast ultrasound overview. Schematic depiction of microbubble administration to enhance the echogenic signal of the blood pool in ultrasound imaging. Continuous infusion of microbubbles at a maintained low mechanical index facilitates visualization of blood flow. A temporary increase in mechanical index results in microbubble destruction, and the refilling of the vascular compartment can be recorded for quantification of replenishment kinetics.

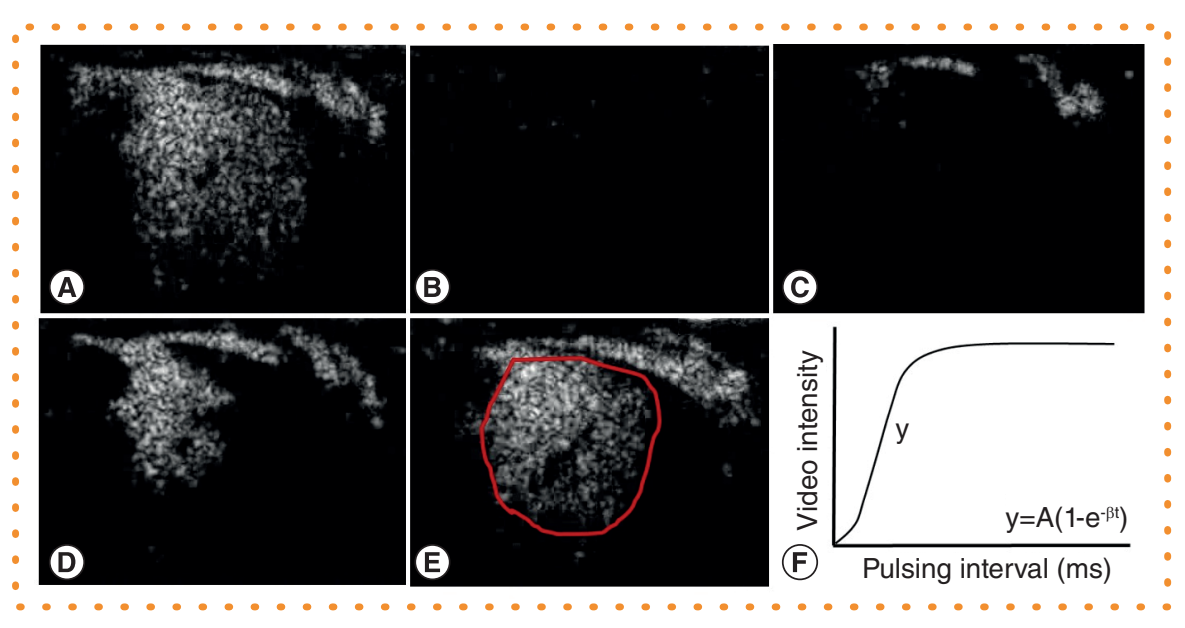

Figure 3. Quantitative analysis of placental contrast enhanced ultrasound imaging. (A-E) Time series of still images from an individual maternal spiral artery perfusing one placental cotyledon in a third trimester nonhuman primate study. (A) Premicrobubble destruction. (B) Microbubbles are 'burst' by a brief increase in the mechanical index. (C) Visualization of refilling of the maternal spiral artery at $1 \mathrm{~s}$ post burst. (D) Perfusion of the intervillous space $4 \mathrm{~s}$ post burst. (E) Replenishment at $10 \mathrm{~s}$ post burst. (F) Example replenishment-kinetic curve indicating calculation of flux rate ( $\beta$ ) from the slope of the curve $(y)$ for a region of interest (red outline in $(E)$ ).

increased cell permeability [33] and holes in the plasma membrane [34]. Several reports utilizing cell culture systems have demonstrated evidence of cell membrane damage following microbubble and ultrasound exposure [35-37] and increased endothelial cell damage via shear stress-induced mechanisms and the production of reactive oxygen species [38]. In vivo data concluded that in the rat myocardium, a low contrast agent dose of $0.1 \mathrm{mg} / \mathrm{kg}$ did no damage, whereas a higher dose of $1.0 \mathrm{mg} / \mathrm{kg}$ increased microvascular injury [36,37]. In a pig model of transcranial contrast-enhanced ultrasound exposure, with and without MRI using gadolinium-based contrast to quantify leakage within the parenchyma, the blood-brain barrier was found to be compromised using a concentration range of $1.5 \times 10^{9}$ to $1.55 \times 10^{10}$ microbubbles/ml [39]. However, the permeability effects noted were only transiently observed at 60 and 90 min post exposure, with 
restoration of blood-brain barrier integrity by $120 \mathrm{~min}$. It has also been suggested that contrast ultrasound exposure may produce a temporary and local rise in temperature [40]. The question remains as to whether these transient localized effects have an impact on overall function and homeostasis, or whether an organ such as the placenta can compensate and regulate function to buffer the impact of these adverse conditions. The history of contrast-enhanced ultrasound use and the safety data that has been generated from pregnant animal model studies will be reviewed next.

\section{Knowledge from animal models \& in vitro studies}

Implementation of contrast imaging in the pregnancy field spans several decades but has been sporadic in nature with relatively poor progression compared with other disciplines. As with most technology development or new application, animal models provide an invaluable experimental resource to determine suitability for transition to use in human beings. Use of contrast agents is permissible, and a multimodal approach, combined with the ability to harvest tissue, allows for comprehensive in vitro assessment of potential adverse impacts of ultrasound and microbubble exposure. Collectively, contrast imaging in a variety of animal species has revealed several key physiological properties of microbubble interactions with the placenta, and impacts on fetal well-being and developmental characteristics of early blood flow in pregnancy.

Lower-order species such as rodents typically provide an opportunity for data generation with studies that can be achieved in a shorter time period and that are affordable in larger cohorts. Using contrast ultrasound, data from studies conducted in rats has demonstrated that microbubbles do not cross the placenta $[41,42]$. Specifically, maternal intravenous administration of Evans blue dye in combination with contrast agent and ultrasound exposure revealed distinct blue dye deposition throughout the placenta but absence of dye in the fetuses delivered 30 min post injection [41]. These findings were robustly assessed using several mechanical index settings, including parameters that exceed typical human exposure in diagnostic imaging. Similarly, a study that used Vevo MicroMarker ${ }^{\circledR}$ bubbles in combination with ultrasound on gestational days 14,17 and 20 , with delivery on day 20 , demonstrated no contrast enhancement in the umbilical vein or fetal tissues. Further assessment demonstrated no macroscopic or histological evidence of adverse effects on fetal weight or placental structure [42]. However, although small animal models provide useful insights, the difference in placental structure can limit aspects of the translational value for human pregnancy. Animal models in higher-order species can be advantageous. In particular, the nonhuman primate has a hemochorial placenta with similar trophoblast invasion characteristics and closely related structure and function to the human placenta $[43,44]$. Earlier work with contrast administration was performed in combination with Doppler ultrasound rather than B-mode imaging. In the pregnant nonhuman primate, maternal administration of Levovist ${ }^{\circledR}$ at a diagnostic dose $(0.1 \mathrm{ml} / \mathrm{kg}$ of $300 \mathrm{mg} / \mathrm{ml})$, a maximal dose $(0.5 \mathrm{ml} / \mathrm{kg}$ of $400 \mathrm{mg} / \mathrm{ml})$ and saline control infusion produced no significant effects on fetal hemodynamics [45], supporting the human reports with this agent $[27,28]$. Similarly, in a sheep model of acute fetal hypoxia that examined the impact of Levovist ${ }^{\circledR}$ to enhance imaging of small fetal vessels with Doppler imaging, no adverse effect of this technology on fetal heart rate or fetal blood flow velocities was shown [46].

Much of the work achieved with animal models and contrast-enhanced ultrasound has demonstrated the feasibility of using this imaging modality to examine aspects of pregnancy and placental function, but quantitative analysis steps have not been rigorously pursued. Third trimester B-mode imaging in rhesus macaques demonstrated maternal perfusion of the intervillous space within $15 \mathrm{~s}$ of bolus administration of phospholipid shelled, perfluoropropane gas-filled microbubbles [47]. In pregnant baboons, time intensity curves were generated from a fixed transducer position with repeated data acquisition (four-times) using both grayscale and Doppler [48] following a contrast bolus. However, replenishment kinetics obtained with constant infusion of contrast agent have only recently been assessed. In a rodent study using SonoVue ${ }^{\circledR}$ microbubbles, which have a phospholipid shell encapsulating sulfur hexafluoride gas (5 $\times 10^{5} \mathrm{MBs} / \mathrm{ml}$ ), contrast-enhanced ultrasound provided high temporal and spatial resolution and revealed real-time sequence and quantitative perfusion parameters in differing regions of the placenta and uterus at different gestational time points [49]. One disadvantage of the rat model is the inability to distinguish between fetal and placental anatomical structures prior to approximately 15 days of gestation [49]; this is not an issue in larger animal models.

\section{Quantitative contrast-enhanced ultrasound}

Studies performed in both nonhuman primate and human subjects have advanced the development and validation of contrast-enhanced ultrasound for the real-time assessment of placental hemodynamics in recent years. The studies summarized in the following all use the contrast agent Definity ${ }^{\circledR}$, which must be activated by $45 \mathrm{~s}$ of vigorous shaking in a Vialmix ${ }^{\circledR}$ to give a suspension containing $1.2 \times 10^{10}$ lipid microspheres and $1.1 \mathrm{mg} / \mathrm{ml}$ of octafluoropropane gas. This formulation is further diluted (1:15) in saline and administered at a continuous infusion rate of $60 \mathrm{ml} / \mathrm{h}$ for replenishment data acquisition in placental imaging, rather than a weight-based dose, which is typical of bolus contrast administration. Definity ${ }^{\circledR}$ microbubbles are commonly used in diagnostic cardiac imaging where the recommended bolus dose is $10 \mu \mathrm{l} / \mathrm{kg}$ of the activated suspension. In toxicology trials conducted by the manufacturer (Lantheus Medical Imaging), use of administered doses 24- and 15-times that of human dosing given in rats and rabbits, respectively, showed no effect on fertility or harm to the fetus.

Longitudinal studies in pregnant macaques have demonstrated that contrast ultrasound can be utilized to quantify increasing microvascular blood flow velocity with advancing gestation, which is perturbed in animal models of dietary manipulation [31,50,51], and viral infection [52]. During imaging sessions, the replenishment kinetics of individually visualized placental cotyledons supplied by a 
maternal spiral artery were calculated in three replicate recordings using optimized and standardized data acquisition parameters that remained consistent throughout each scan. Data were methodically acquired by systematically moving the transducer across the abdomen to visualize and record from each spiral artery in turn. Calculation of absolute blood volume and blood flow is possible using the analysis software $\mathrm{Narnar}^{\circledR}$ but with the caveat that the equations were generated for application to a typical capillary network, which is not what is found in the complex microvasculature of the placenta (Figure 1). Thus, data from these studies have been reported as flux rate, which provides an estimate of vascular impendence, or microvascular blood flow velocity, in the intervillous space [53]. Importantly, perturbations in flux rate were detected when Doppler measures of impedance (the Pulsatility Index) in the uterine artery and umbilical artery were not detectable $[31,50,51]$, suggesting increased sensitivity of contrast ultrasound for assessment of placental dysfunction when compared with traditional measures of placental function by Doppler ultrasound.

The nonhuman primate studies have been performed in pregnancies utilized for fetal studies where placental tissue was harvested following cesarean section delivery in the third trimester, and in continuing pregnancies where the animals deliver naturally and no placental tissue was available. Importantly, contrast-enhanced ultrasound use in mid and late gestation, in addition to very early implantation assessments, resulted in no pregnancy loss and normal term delivery of healthy infants [51,54]. Throughout each ultrasound, the animals underwent continuous physiological monitoring of heart rate, blood pressure, respiratory rate and temperature: no deviations from normal parameters have been reported and no adverse reactions to contrast agent administration have occurred in this animal model.

\section{Contrast-enhanced ultrasound in pregnant human subjects}

Performing contrast-enhanced ultrasound in human subjects is constrained by the cautious approach surrounding the use of administered agents in pregnant women, and thus these experimental quantitative analysis studies have been limited to noncontinuing pregnancies with ultrasound performed in the first trimester immediately prior to termination [53,55]. These studies have revealed some important findings of perfusion characteristics in early placental development. Specifically, early imaging of the whole placenta demonstrated regional perfusion differences that do not correspond to central versus peripheral cotyledon location but instead show the heterogeneous nature of the placenta [53]. In addition, the use of this in vivo imaging modality, which has the sensitivity and resolution to facilitate visualization of microvascular filling of the intervillous space, revealed new evidence of first trimester flow earlier in pregnancy than previously reported, as early as 6 weeks of gestation and consistently from 7 weeks onward [55]. This study also demonstrated an increase in spiral artery flux rate at the end of the first trimester, suggesting that additional mechanisms beyond spiral artery remodeling contribute to the regulation of early placental blood flow in humans [55]. The hypothesis that radial arteries contribute to early flow regulation is supported by murine studies using placental microcomputed tomography [56,57], and a computational modeling approach that predicted the radial arteries to be the rate-limiting step in regulating maternal blood flow to the placental intervillous space $[58,59]$.

In a subset of the human samples exposed to first trimester contrast ultrasound, post-procedure placental tissue was obtained for histological assessment of tissue integrity. Importantly, there was no evidence of microvascular hemorrhage that could result from cavitation, no lodging of microbubbles in the intervillous space and a normal appearance of the microvillous membrane of the syncytiotrophoblast by ultrastructural electron microscopy analysis [53]. Similarly, collection of placental tissue from timed deliveries in the macaque studies facilitated in vitro assessment of placental tissue integrity with and without exposure to ultrasound with microbubbles, and demonstrated no induction of apoptosis, complement-mediated signaling or nitrative stress [53]. Taken together, these reports provide reassuring outcomes to suggest that placental tissue integrity is not compromised by the use of microbubbles in combination with ultrasound exposure and that placental function is not altered following the use of this imaging modality. As a follow-up to the initial human work, there is an ongoing clinical study in France in which the primary objective is to use both contrast-enhanced ultrasound and 3D power Doppler angiography to assess perfusion at 8,11 and 13 weeks gestation in noncontinuing pregnancies [60].

\section{Practical considerations for the clinical implementation of contrast-enhanced ultrasound}

Early in pregnancy, it is possible to capture the entire placenta in one field of view, whereas closer to term, the placental size exceeds the optimal range for acoustic imaging with the available transducer and thus requires methodical scanning across the uterus. Placental position, maternal body composition and other anatomical factors could impede the ability to adequately capture the placenta. Importantly, a strong correlation has been demonstrated between data obtained from the same maternal spiral artery sources in the sagittal and coronal views in human subjects, which is necessary for the validation and reproducibility of this methodology [55]; this is an important consideration for an application that would be used by multiple users across a wide range of clinical settings. The duration of each contrast ultrasound examination averaged $20 \mathrm{~min}$, during which time replenishment kinetics from $\sim 6$ to 10 spiral arteries were recorded in three replicate sites per source, and baseline measurements were made in the maternal uterine artery and brachial artery of the noncatheterized arm. These baseline assessments of steady state flow (no microbubble destruction) are necessary if calculation of absolute blood flow and volume is intended in later analysis.

\section{Advanced microbubble applications}

Reluctance to implement contrast-enhanced ultrasound in obstetrics has not extended to other medical fields where the potential of this technology has been well recognized, and significant advancements to its application have been made. Specifically, development 
of specialized microbubble formulations and composition has provided new possibilities for diagnostic imaging and interventional therapeutics. For example, incorporation of specialized core content, or modifications such as ligand attachment to the outer surface of microbubbles [17,21], provides a mechanism for molecular imaging [61], and targeted drug and gene delivery [62], which is achieved when the microbubbles are destroyed within the beam of the ultrasound transducer in a site-specific manner [21,30]. This extensive and promising research area is beyond the scope of the current review but offers additional future opportunities for in vivo pregnancy diagnostics.

\section{Future perspective}

This review has provided a summation of the benefits and the knowledge gained using contrast-enhanced ultrasound to date. Prior to implementing this imaging modality into routine obstetric management, several important considerations may still need to be evaluated, and utilization in ongoing pregnancies will require further human studies. The challenge remains in alleviating safety concerns to trial the use of contrast agents for the assessment of placental function in pregnant volunteers. Promisingly, data from animal models has shown no evidence of placental tissue damage or altered function, in addition to demonstrating good diagnostic potential and the ability to detect pregnancies at risk for placental dysfunction early in gestation using this imaging modality. Taken together, a compilation of studies suggests that implementing the use of this method to generate clinically relevant quantitative real-time data in continuing human pregnancies is merited. Early detection will guide further diagnostic assessment requirements and facilitate intervention with the ultimate goal of improving pregnancy outcome.

\section{Author contributions}

VHJ Roberts reviewed the literature, prepared the figures and wrote the manuscript. AE Frias provided financial support and edited the manuscript.

\section{Financial \& competing interests disclosure}

Funding support was provided by the National Institute of Health (R21 HD 076265), the NICHD Human Placenta Project (U01 HD 087182 and R01 HD 086331; AE Frias), and Seed funding from the Oregon Health and Science University Knight Cardiovascular Institute Center for Developmental Health and the Struble Foundation. The authors have no other relevant affiliations or financial involvement with any organization or entity with a financial interest in or financial conflict with the subject matter or materials discussed in the manuscript apart from those disclosed.

No writing assistance was utilized in the production of this manuscript.

\section{Open access}

This work is licensed under the Attribution-NonCommercial-NoDerivatives 4.0 Unported License. To view a copy of this license, visit http://creativecommons.org/licenses/by-nc-nd/4.0/

\section{References}

1. Kliman HJ. Uteroplacental blood flow. The story of decidualization, menstruation, and trophoblast invasion. Am. J. Pathol. 157(6), 1759-1768 (2000).

2. Moll W. Structure adaptation and blood flow control in the uterine arterial system after hemochorial placentation. Eur. J. Obstet. Gynecol. Reprod. Biol. 110(Suppl. 1), S19-S27 (2003).

3. Kidron D, Bernheim J, Aviram R. Placental findings contributing to fetal death, a study of 120 stillbirths between 23 and 40 weeks gestation. Placenta 30(8), 700-704 (2009).

4. Roberts DJ, Post MD. The placenta in pre-eclampsia and intrauterine growth restriction. J. Clin. Pathol. 61(12), 1254-1260 (2008).

5. Guttmacher AE, Maddox YT, Spong CY. The Human Placenta Project: placental structure, development, and function in real time. Placenta 35(5), 303-304 (2014).

6. Thornton KL. Principles of ultrasound. J. Reprod. Med. 37(1), 27-32 (1992).

7. Merritt CR. Doppler US: the basics. Radiographics 11(1), 109-119 (1991).

8. Moorthy RS. Doppler ultrasound. Med. J. Armed Forces India. 58(1), 1-2 (2002).

9. Ozkaya U, Ozkan S, Ozeren S, Corakci A. Doppler examination of uteroplacental circulation in early pregnancy: can it predict adverse outcome? J. Clin. Ultrasound 35(7), 382-386 (2007).

10. Zhong Y, Tuuli M, Odibo AO. First-trimester assessment of placenta function and the prediction of preeclampsia and intrauterine growth restriction. Prenat. Diagn. 30(4), 293-308 (2010).

11. Collins SL, Birks JS, Stevenson GN, Papageorghiou AT, Noble JA, Impey L. Measurement of spiral artery jets: general principles and differences observed in small-for-gestational-age pregnancies. Ultrasound Obstet. Gynecol. 40(2), 171-178 (2012).

12. Collins SL, Stevenson GN, Noble JA, Impey L. Developmental changes in spiral artery blood flow in the human placenta observed with colour Doppler ultrasonography. Placenta 33(10), 782-787 (2012).

13. Stevenson GN, Collins SL, Welsh AW, Impey LW, Noble JA. A technique for the estimation of fractional moving blood volume by using three-dimensional power Doppler US. Radiology 274(1), 230-237 (2015).

14. Stevenson GN, Noble JA, Welsh AW, Impey L, Collins SL. Automated visualization and quantification of spiral artery blood flow entering the first-trimester placenta, using 3-D power Doppler ultrasound. Ultrasound Med. Biol. 44(3), 522-531 (2018).

15. Correas JM, Bridal L, Lesavre A, Mejean A, Claudon M, Helenon O. Ultrasound contrast agents: properties, principles of action, tolerance, and artifacts. Eur. Radiol. 11(8), 1316-1328 (2001).

16. Gramiak R, Shah PM. Echocardiography of the aortic root. Invest. Radiol. 3(5), 356-366 (1968).

17. Sirsi S, Borden M. Microbubble compositions, properties and biomedical applications. Bubble Sci. Eng. Technol. 1(1-2), 3-17 (2009).

18. Appis AW, Tracy MJ, Feinstein SB. Update on the safety and efficacy of commercial ultrasound contrast agents in cardiac applications. Echo. Res. Pract. 2(2), R55-R62 (2015).

19. Lindner JR, Song J, Jayaweera AR, Sklenar J, Kaul S. Microvascular rheology of Definity microbubbles after intra-arterial and intravenous administration. J. Am. Soc. Echocardiogr. 15(5), 396-403 (2002).

20. Lepper W, Belcik T, Wei K, Lindner JR, Sklenar J, Kaul S. Myocardial contrast echocardiography. Circulation 109(25), 3132-3135 (2004).

21. Dijkmans PA, Juffermans LJ, Musters RJ et al. Microbubbles and ultrasound: from diagnosis to therapy. Eur. J. Echocardiogr. 5(4), 245-256 (2004).

22. Quaia E. Assessment of tissue perfusion by contrast-enhanced ultrasound. Eur. Radiol. 21(3), 604-615 (2011). 
23. Kaufmann BA, Wei K, Lindner JR. Contrast echocardiography. Curr. Probl. Cardiol. 32(2), 51-96 (2007).

24. Main ML, Goldman JH, Grayburn PA. Thinking outside the "box"-the ultrasound contrast controversy. J. Am. Coll. Cardiol. 50(25), 2434-2437 (2007).

25. Wei K, Mulvagh SL, Carson L et al. The safety of deFinity and Optison for ultrasound image enhancement: a retrospective analysis of 78,383 administered contrast doses. J. Am. Soc. Echocardiogr. 21(11), 1202-1206 (2008).

26. Liu YN, Khangura J, Xie A et al. Renal retention of lipid microbubbles: a potential mechanism for flank discomfort during ultrasound contrast administration. J. Am. Soc. Echocardiogr. 26(12), 1474-1481 (2013).

27. Orden MR, Leinonen M, Kirkinen P. Contrast-enhanced ultrasonography of uteroplacental circulation does not evoke harmful CTG changes or perinatal events. Fetal Diagn. Ther. 15(3), $139-145(2000)$.

28. Denbow ML, Welsh AW, Taylor MJ, Blomley MJ, Cosgrove DO, Fisk NM. Twin fetuses: intravascular microbubble US contrast agent administration-early experience. Radiology 214(3), 724-728 (2000)

29. De Jong N, Bouakaz A, Frinking P. Basic acoustic properties of microbubbles. Echocardiography 19(3), 229-240 (2002).

30. Villanueva FS. Getting good vibes: the therapeutic power of microbubbles and ultrasound. JACC Cardiovasc. Imaging 5(12), 1263-1266 (2012),

31. Salati JA, Roberts VHJ, Schabel MC et al. Maternal high-fat diet reversal improves placental hemodynamics in a nonhuman primate model of diet-induced obesity. Int. J. Obes. (Lond.) 43(4), 906-916 (2019).

32. Wei K, Jayaweera AR, Firoozan S, Linka A, Skyba DM, Kaul S. Quantification of myocardial blood flow with ultrasound-induced destruction of microbubbles administered as a constant venous infusion. Circulation 97(5), 473-483 (1998).

33. Wamel JET, Bouakaz A, Houtgraaf J, Cate FJ, de Jong N. Effects of diagnostic ultrasound parameters on molecular uptake and cell viability. Proceedings of the International Ultrasonics Symposium. Munich, Germany (8-11 October 2002).

34. Stride E, Saffari N. On the destruction of microbubble ultrasound contrast agents. Ultrasound Med. Biol. 29(4), 563-573 (2003).

35. Miller DL, Quddus J. Sonoporation of monolayer cells by diagnostic ultrasound activation of contrast-agent gas bodies. Ultrasound Med. Biol. 26(4), 661-667 (2000).

36. Kobayashi N, Yasu T, Yamada S et al. Endothelial cell injury in venule and capillary induced by contrast ultrasonography. Ultrasound Med. Biol. 28(7), 949-956 (2002).

37. Kobayashi N, Yasu T, Yamada S et al. Influence of contrast ultrasonography with perflutren lipid microspheres on microvessel injury. Circ. J. 67(7), 630-636 (2003).

38. Basta G, Venneri L, Lazzerini G et al. In vitro modulation of intracellular oxidative stress of endothelial cells by diagnostic cardiac ultrasound. Cardiovasc. Res. 58(1), 156-161 (2003).

39. Xie F, Boska MD, Lof J, Uberti MG, Tsutsui JM, Porter TR. Effects of transcranial ultrasound and intravenous microbubbles on blood brain barrier permeability in a large animal model. Ultrasound Med. Biol. 34(12), 2028-2034 (2008).

40. $\mathrm{Wu}$ J. Temperature rise generated by ultrasound in the presence of contrast agent. Ultrasound Med. Biol. 24(2), 267-274 (1998).

41. Hua X, Zhu LP, Li R, Zhong H, Xue YF, Chen ZH. Effects of diagnostic contrast-enhanced ultrasound on permeability of placental barrier: a primary study. Placenta 30(9), 780-784 (2009).

42. Arthuis CJ, Novell A, Escoffre JM, Patat F, Bouakaz A, Perrotin F. New insights into uteroplacental perfusion: quantitative analysis using Doppler and contrast-enhanced ultrasound imaging. Placenta 34(5), 424-431 (2013).

43. Panigel M, James AE Jr, Siegel M, Donner MW. Radionuclide and angiographic studies of placental circulation in man and rhesus monkey. Eur. J. Obstet. Gynecol. Reprod. Biol. 5(5), 251-262 (1975).

44. Ramsey EM, Houston ML, Harris JW. Interactions of the trophoblast and maternal tissues in three closely related primate species. Am. J. Obstet. Gynecol. 124(6), 647-652 (1976).

45. SchmiedI UP, Komarniski K, Winter TC et al. Assessment of fetal and placental blood flow in primates using contrast enhanced ultrasonography. J. Ultrasound Med. 17(2), 75-80 discussion 81-82 (1998).

46. Grussner S, Klingmuller V, Bohle R. [Increased signal intensity of velocity measurements in duplex sonography by using the contrast agent levovist: a prospective, randomized study in a fetal sheep model]. Rofo. 176(1), 91-97 (2004).

47. Ragavendra N, Tarantal AF. Intervillous blood flow in the third trimester gravid rhesus monkey (Macaca mulatta): use of sonographic contrast agent and harmonic imaging. Placenta 22(2-3), 200-205 (2001)

48. Barth WH, McCurnin DC, Dee Carey K, Hankins GD. Contrast sonography, video densitometry and intervillous blood flow: a pilot project. Placenta 27(6-7), 719-726 (2006).

49. Zhou YJ, Yuan ML, Li R, Zhu LP, Chen ZH. Real-time placental perfusion on contrast-enhanced ultrasound and parametric imaging analysis in rats at different gestation time and different portions of placenta. PLOS ONE 8(4), e58986 (2013).

50. Kuo K, Roberts VHJ, Gaffney J et al. Maternal high-fat diet consumption and chronic hyperandrogenemia are associated with placental dysfunction in female rhesus macaques. Endocrinology 160(8), 1937-1949 (2019).

51. Roberts VHJ, Lo JO, Lewandowski KS et al. Adverse placental perfusion and pregnancy outcomes in a new nonhuman primate model of gestational protein restriction. Reprod. Sci. 25(1), $110-119$ (2018).

52. Hirsch AJ, Roberts VHJ, Grigsby PL et al. Zika virus infection in pregnant rhesus macaques causes placental dysfunction and immunopathology. Nat. Commun. 9(1), 263 (2018).

53. Roberts VH, Lo JO, Salati JA et al. Quantitative assessment of placental perfusion by contrast-enhanced ultrasound in macaques and human subjects. Am. J. Obstet. Gynecol. 214(3), 369.e1-369.e8 (2016).

54. Keator CS, Lindner JR, Belcik JT, Bishop CV, Slayden OD. Contrast-enhanced ultrasound reveals real-time spatial changes in vascular perfusion during early implantation in the macaque uterus. Fertil. Steril. 95(4), 1316-1321.e3 (2011).

55. Roberts VHJ, Morgan TK, Bednarek P et al. Early first trimester uteroplacental flow and the progressive disintegration of spiral artery plugs: new insights from contrast-enhanced ultrasound and tissue histopathology. Hum. Reprod. 32(12), 2382-2393 (2017).

56. Cahill LS, Rennie MY, Hoggarth J et al. Feto- and utero-placental vascular adaptations to chronic maternal hypoxia in the mouse. J. Physiol. 596(15), 3285-3297 (2018).

57. Rennie MY, Whiteley KJ, Adamson SL, Sled JG. Quantification of gestational changes in the uteroplacental vascular tree reveals vessel specific hemodynamic roles during pregnancy in mice. Biol. Reprod. 95(2), 43 (2016)

58. Clark AR, James JL, Stevenson GN, Collins SL. Understanding abnormal uterine artery Doppler waveforms: a novel computational model to explore potential causes within the uteroplacental vasculature. Placenta 66, 74-81 (2018)

59. James JL, Saghian R, Perwick R, Clark AR. Trophoblast plugs: impact on utero-placental haemodynamics and spiral artery remodelling. Hum. Reprod. 33(8), 1430-1441 (2018).

60. Bertholdt C, Eszto ML, Tournier M et al. Assessment of uteroplacental vascularisation in early first-trimester pregnancy with contrast-enhanced ultrasound and 3D power Doppler angiography: protocol for a prospective, cross-sectional, multicentre and non-randomised open study ("HOPE Study"). BMJ Open 9(9), e030353 (2019).

61. Lindner JR, Sinusas A. Molecular imaging in cardiovascular disease: which methods, which diseases? J. Nucl. Cardiol. 20(6), 990-1001 (2013).

62. Xie A, Belcik T, Qi Y et al. Ultrasound-mediated vascular gene transfection by cavitation of endothelial-targeted cationic microbubbles. JACC Cardiovasc. Imaging 5(12), 1253-1262 (2012). 